\title{
Efficacy of static magnetic field for pain of adjuvant arthritis rats
}

\author{
Shigeyuki Kanai ${ }^{1}$, Norimasa Taniguchi ${ }^{1,2}$ \\ ${ }^{1}$ Department of Physical Therapy, Faculty of Health Science, Kansai University of Health Science, Osaka, Japan \\ ${ }^{2}$ Research Section for Magnetics, Product Development Department, Development Division, PIP Co, Ltd, Osaka, Japan \\ Email: n-taniguchi@pipjapan.co.jp, kanai@kansai.ac.jp
}

Received 29 June 2012; revised 30 July 2012; accepted 13 August 2012

\begin{abstract}
Rats suffering from adjuvant arthritis (AA) were used to examine the effect of a static magnetic field (SMF) upon pain relief. Rats were divided into SMFtreated AA rats, non-SMF treated AA rats and control rats. Following SMF stimulation, we measured blood flow volume in the paw and then reactive speed response to thermal stimulation. The AA groups exhibited significantly lower blood volume and reactivity to thermal stimulation compared to the control group. Compared to non-SMF, SMF exhibited increased blood flow volume in both the tail and paw, along with an increased reactive speed response to thermal stimulation. Our findings suggest that an improved of blood flow and reactive speed response, induced by SMF, appears to be effective for the relief of pain induced by chronic inflammation.
\end{abstract}

Keywords: Static Magnetic Field (SMF); Adjuvant Arthritis (AA); Blood Flow; Reactive Speed Response

\section{INTRODUCTION}

Over recent years, the biological response of exposure to static magnetic fields (SMF) has become the focus of much debate, particularly in terms of clinical relevance and the potential for adverse effects. Previous experiments have shown that local SMF stimulation can be beneficial for pain for the treatment of pain and nerve regeneration [1], imflammation [2], blood flow [3], cutaneous microcirculation [4], blood pressure [5-7] and united fractures [8-10].

SMF, generated by small cylindrical Neodymium-ironboron rare earth magnets and ferrite magnets, have already been used to provide pain relief for low back pain and knee pain arising from ischemic conditions of the blood microcirculation [11-13]. In Japan, SMF generated by small, cylindrical Ferrite magnets have been used to provide pain relief from neck, shoulder and lower back pain [14-16]. These magnetotherapies have been shown to be clinically effective.

Adjuvant arthritis (AA) in the rat is an excellent model of acute periarticular proliferative synovitis and is induced by a single intradermal injection of tubercle bacillus to the hind paw [17]. As one of the main experimenttal models of arthritis, AA rats have been used to evaluate the efficacy of several drugs at many institutions, with particular emphasis placed upon anti-inflammatory drugs [18].

In the present study, AA rats were left untreated for a prolonged period in order to develop chronic pain models. The influence of SMF treatment upon the blood flow in the tail and paw, along with the reactive speed response (pain relief), was studied using laser Doppler perfusion imaging and the Plantar test.

\section{MATERIALS AND METHODS}

\subsection{Study Animals and Environmental Conditions}

Female Sprague-Dawley (SD) rats (age: 5 weeks, body weight: approximately $150 \mathrm{~g}$ ) were purchased from Japan Crea Co. Ltd. (Shizuoka, Japan). Animals were housed individually in cages and were kept in a room maintained at a temperature of $23.0^{\circ} \mathrm{C} \pm 1.0^{\circ} \mathrm{C}$ with a relative humidity (RH) of $55.0 \% \pm 5.0 \%$ and a $12 \mathrm{~h} / 12 \mathrm{~h}$ light/dark cycle.

Solid rodent chow and tap water were offered ad libitum. Animals were conditioned to these conditions for one week and individuals showing favorable growth patterns were selected for further studies.

This research project was recognized by the University Ethics Committee for animal studies. Animals were culled by an overdose of anaesthesia and the femur removed for analysis.

\subsection{Experimental Animals}

Sixty female SD rats (age: 6 weeks, body weight: approximately $160 \mathrm{~g}$ ) were divided into 3 groups. In Group I (6 animals) and Group II (6 animals), we injected $0.5 \mathrm{ml}$ 
of incomplete Freund's Adjuvant (Wako co., Ltd.) into the left hind foot in order to induce AA. Injected animals were then maintained for a further 8 weeks in order to develop a chronic pain model. Immediately following this 8 weeks induction period, Group I was exposed to SMF treatment for a further 4 weeks (up to week 12 following the onset of AA). Group II (the non-SMF treated AA rats) were not exposed to SMF treatment. Group III (control rats, $n=6$ ) were maintained without any treatment for a total period of 12 weeks (Figure 1).

\subsection{Conditions of Exposure to SMF}

The SMF exposure device (Length: $300 \mathrm{~mm}$; Height: 130 mm; Width: 440 mm, PIP Co., Ltd., Osaka, Japan) was composed of a pair of rectangular magnetic plates, externally placed parallel to and at the shortest distance of $-1 \mathrm{~cm}$ from two sides of a transparent cage (Length: $340 \mathrm{~mm}$, Height: $180 \mathrm{~mm}$, Width: $400 \mathrm{~mm}$ ) (Figures 1(A), (B)). The mean flux density at the center of a cage and the magnetic surface was $30 \mathrm{mT}$ (range $=20-80 \mathrm{mT}$ ) and $200 \mathrm{mT}$, respectively (Figure 1(C)).

For Group I, the SMF exposure device was fixed on the left and right sides of the cage and each animal was exposed to the magnetic field continuously for a period of 4 weeks. For Group II, a magnetic flux density of $0 \mathrm{mT}$ was delivered, to act as a control.

\subsection{Measurement of Physiological Parameters}

\subsubsection{Blood Flow}

Animals were first allowed to acclimate to their environment (temperature $=23.0^{\circ} \mathrm{C} \pm 1.0^{\circ} \mathrm{C}$, relative humidity $=55.0 \% \pm 5.0 \%$; no air movement) for a period of 15 minutes. Blood flow was then measured in the tail and hind paw of conscious animals using a laser Doppler perfusion Imager (LDI) (PeriScan PIM II; Sweden PERIMED Co. Ltd.).

To avoid the possible influence of coat hair, we considered tail and paw blood flow as an indicator of the peripheral circulation $[17,19]$. Blood flow in the tail and hind paw was measured before and after exposure to SMF over a 4 weeks period (between 14 weeks and 18 weeks post-induction) in Group I, and at the same time points in Group II and Group III.

The effects of perfusion were measured in the tail and hind paw with the LDI. This non-invasive technique generates a two-dimensional representation of tissue perfusion based upon the concentration and speed of circulating erythrocytes in the articular microvasclature. All perfusion images were analyzed by LDI processing software (PERIMED Co. Ltd., Sweden) and average flux for the
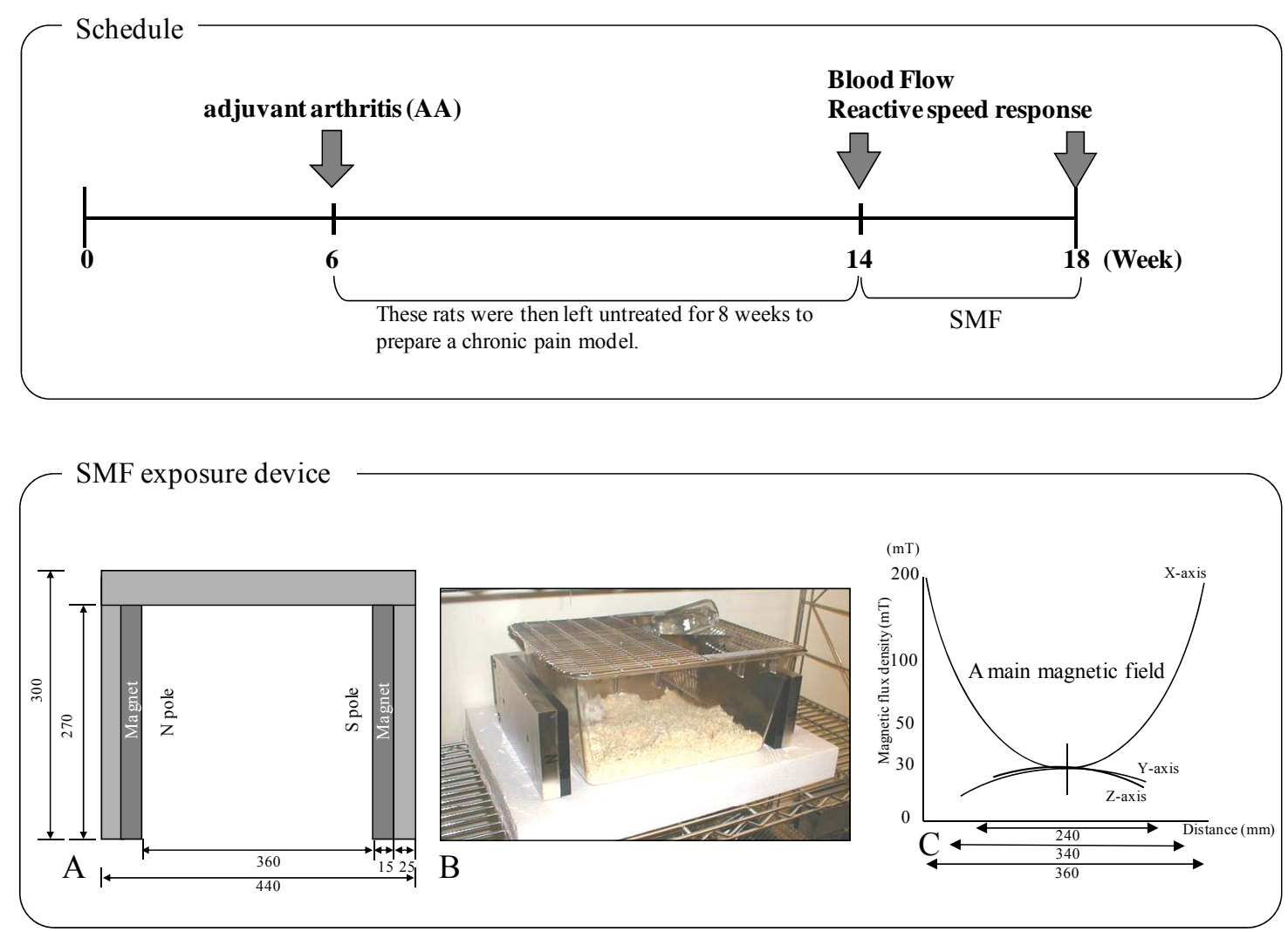

Figure 1. Schedule \& SMF exposure device [Schematic diagram of magnet show its top (A) and front (B) plan view. The mean magnetic force at the center of the cage was $30 \mathrm{mT}(\mathrm{C})]$. 
anteromedical aspect of the joint calculated and expressed in arbitrary units.

\subsubsection{Pain-Related Behaviour (Reactive Speed Response)}

The reactive speed response of the paw to thermal stimulation was measured using a Reactive Speed Response. Planter Test (Ugo Basile Planter test 7370, UGO BASILE, Italy) [19]. The equipment used for this method consists of a movable light generator which is manipulated under a glass pane by the operator. The rats are placed onto the glass pane in a 3-compartment plexiglass enclosure. The withdrawal latency of the animal's paw is measured automatically by the equipment [20].

The reactive speed response to thermal stimulation was used as an indicator of the peripheral circulation. Thermal stimulation was measured before and after exposure to SMF for a period of 4 weeks (between 14 weeks and 18 weeks post-induction) in Group I, and at the same time points in Group II and Group III.

For higher objectivity and more precise comparability, we also assessed the time difference of withdrawal latencies between the animals measured before and after the experiment.

The absolute values of withdrawal latencies varied from 6 to 10 seconds in the Planter Test.

\subsection{Data Analyses}

Data obtained for each group were expressed as the mean \pm standard error. The significance of differences observed between experimental groups was assessed using the Wilcoxon sum-rank test. Significance level was set at $P<0.05$.

\section{RESULTS}

\subsection{Blood Flow before and after Exposure to SMF}

Tail and paw blood flow was significantly lower in AA rats (Group I and Group II) than control rats (Group III) when analysed prior to the exposure of Group I to SMF at the 8 weeks timepoint. After 4 weeks exposure of Group I to SMF (between the 14 weeks and 18 weeks timepoint), it was found that the blood flow in the tail and hind foot of the SMF-treated AA rats (Group I) was significantly higher than that of the non-SMF treated AA rats (Group II). The change in blood flow latency between the beginning and end of the experiment was as follows: AA + SMF (Group I): $0.93 \pm 0.02 \rightarrow 1.11 \pm$ 0.03 ; AA (Group II): $0.92 \pm 0.03 \rightarrow 0.82 \pm 0.04$; Control (Group III): $1.12 \pm 0.03 \rightarrow 1.15 \pm 0.05$ ). There was no significant difference in blood flow (tail or hind paw) when compared between Group I and Group III at the 18 weeks timepoint (Figure 2).

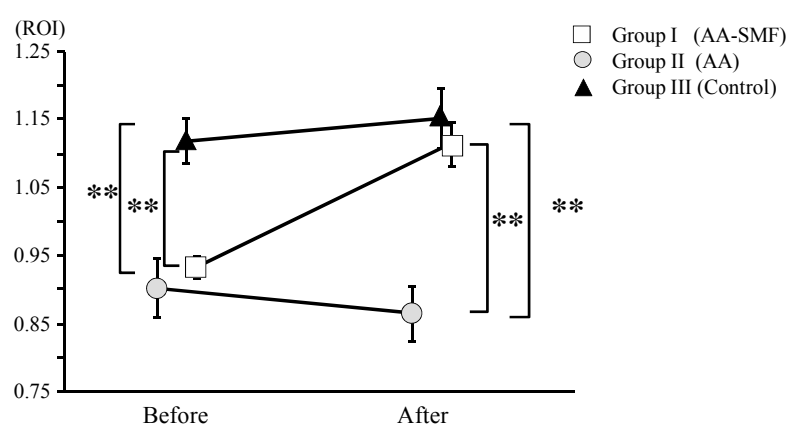

Figure 2. Pain-related score in the tail and hind paw of AA and normal rats before and after 12-week exposure to the SMF. Group II and Group III were not exposed to the SMF. The results are expressed as mean \pm SEM. $* P<0.05$; $* * P<0.01$.

\subsection{Pain-Related Score before and after Exposure to SMF}

The reactive speed response (Planter Test) was significantly slower in the AA rats (Group I and Group II) than in the control rats (Group III) when tested before the exposure of Group I to SMF at the 8 weeks timepoint. Following exposure of Group I to SMF for 4 weeks (between weeks 14 and 18), we found that the reactive speed response of the SMF-treated AA rats (Group I) was significantly faster than that of the non-SMF treated AA rats (Group II). The change of planter test latency when compared before and after the experiment was as follows: AA + SMF (Group I): $8.5 \pm 0.3 \rightarrow 7.2 \pm 0.4$; AA (Group II): $8.1 \pm 0.6 \rightarrow 9.6 \pm 0.5$; Control (Group III): $5.3 \pm 0.2$ $\rightarrow 6.4 \pm 0.3$. There was no significant difference in reactive speed response when compared between Group I and Group III at the 18 weeks timepoint (Figure 3).

\section{DISCUSSION}

AA has been widely studied and is used as a model of experimental arthropathy in the evaluation of drugs, with particular emphasis upon anti-inflammatory agents $[18$, $[21,22]$.

Rheumatic arthritis is often accompanied by ankylosing deformity and coldness of joints, and it is currently postulated that AA rats that have not been treated for a prolonged period can be used as an experimental model of abnormal cold feeling. It is also known that inflammation of one knee can produce a symmetrical response in the other knee, as well as inducing increased pro-inflammatory neuropeptide levels within the synovial fluid of the contralateral knee resulting in modification of contralateral basal blood composition [23].

In the present study, we maintained AA rats without treatment in order to utilize these animals as models of chronic ischemic pain. The mechanism responsible for the development of rat AA involves autoimmunity cross-reaction, in which $\mathrm{T}$ lymphoids, that are sensitized 


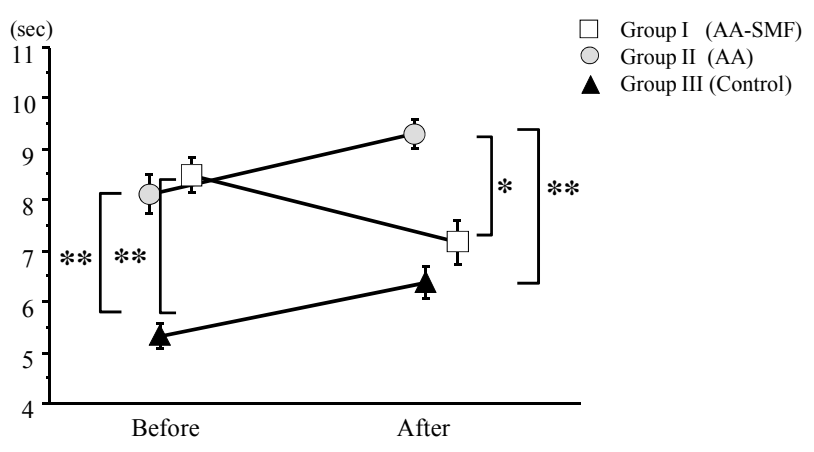

Figure 3. Blood flow of AA and normal rats before and after 12-week exposure to the SMF. Group II and Group III were not exposed to the SMF. The results are expressed as mean \pm SEM. ${ }^{*} P<0.05 ; * * P<0.01$.

to tubercle bacillus, cross-react with a protein in the articular cartilage of the rat, thus causing arthritis [18].

However, the pathology of this model may not be the same as that of human rheumatic arthritis, despite the fact that similar symptoms develop. In brief, although redness and swelling in a variety of joints worsen $2-3$ weeks after the induction of AA, these symptoms subsequently resolve, leaving articular ankylosing deformity. Histologically, both chronic granulomatous inflammation and acute inflammation associated with inflammatory cell infiltration have been noted in rat AA [21,22]. The disease does not become a chronic condition.

Previous studies have investigated the effect of SMF upon animals that had remained untreated for 6 months after the induction of AA [24]. Since the tail surface temperature, locomoter activity and bone mineral density of AA animals significantly increased after prolonged exposure to the SMF for 12 weeks, it was suggested that exposure to SMF resulted in improvements to the peripheral circulation. Consequently, it is vital that these observations are expanded by basic research using a specific pathophysiological adjuvant arthritis model rat. This approach is necessary in order to elucidate the precise mechanism by which SMF results in pain relief in various painful conditions such as ischemic or inflammatory pain.

In rats, we have previously reported that the results of the planter test, blood flow, and activity of AA rats treated with SMF were significantly increased compared to conventional Electro-Acupuncture Therapy (muscleEAT), a widely employed perspective effect for pain [17].

The precise mechanism of action of SMF and its effect upon pain remain unclear, although previous studies have shown that exposure to a magnetic force resulted in the increased release of acetylcholine from cholinergic vasodilator nerve endings by inhibiting the effect of cholinesterase, thus resulting in vasodilation. Takeshige et al. [25] provided evidence to suggest the mechanism under- lying the pain reduction effect caused by more general magnetotherapeutic devices. These authors reported that exposure to magnetic fields may stimulate cholinergic nerves by suppressing cholinesterase activity and that the resultant release of acetylcholine dilated vascular smooth muscles and elevated blood flow.

In the present study, we found that after exposure to SMF, the results of the planter test of AA rats changed to levels observed in normal rats. We also observed a significant decrease in overall reactive speed response (painrelated) in the SMF-treated AA rat, along with an increase in blood flow. Moreover, Pekarkova et al. [20] further reported that the plantar test is a useful technique for pain relief. Consequently, we postulate that the SMFinduced increase in blood flow observed in the AA rats described in the present study was most likely due to the removal of pain rather than to the induction of stress.

Further basic research, using a specific pathophysiological animal model are necessary in order to fully elucidate the precise manner in which SMF relieves pain in a variety of painful conditions, including ischemic pain and inflammatory pain. As complementary and alternative medicine continues to expand, there is increasing interest in the potential therapeutic use of SMF for therapeutic uses. Existing literature already describes the analgesic actions of SMF. Furthermore, a clinical study has shown that SMF treatment successfully relieved pain in patients suffering from neck shoulder pain and muscle fatigue as a direct result of ischemic conditions in the microcirculation. We consider it important to combine the application of SMF with Western medicine or exercise therapy.

\section{REFERENCES}

[1] Veliks, V., Ceihnere, E., Svkis, I. and Aivars, J. (2004) Static magnetic field influence on rat brain function detected by heart rate monitoring. Bioelectromagnetics, 25, 211-215. doi:10.1002/bem.10186

[2] Morris, C.E. and Skalak, T.C. (2008) Magnetic therapy for edema in inflammation: A physiological assessment. American Journal of Physiology-Heart and Circulatory Physiology, 294, H50-H57. doi:10.1152/ajpheart.00529.2007

[3] Gmitrov, J., Ohkubo, C. and Okano, H. (2002) Effect of $0.25 \mathrm{~T}$ static magnetic field on microcirculation in rabbits. Bioelectromagnetics, 23, 224-229. doi:10.1002/bem.10007

[4] Xu, S., Okano, H. and Ohkubo, C. (2001) Acute effects of whole-body exposure to static magnetic fields and $50-\mathrm{Hz}$ electromagnetic fields on muscle microcirculation in anesthetized mice. Bioelectrochemistry, 53, 127-135. doi:10.1016/S0302-4598(00)00120-3

[5] Okano, H. and Ohkubo, C. (2005) Effects of neck exposure to $5.5 \mathrm{mT}$ static magnetic field on pharmacologically modulated blood pressure in conscious rabbits. Bioelec- 
tromagnetics, 26, 469-480. doi:10.1002/bem.20115

[6] Okano, H. and Ohkubo, C. (2005) Exposure to a moderate intensity static magnetic field enhances the hypotensive effect of a calcium channel blocker in spontaneously hypertensive rats. Bioelectromagnetics, 26, 611-623. doi:10.1002/bem.20144

[7] Okano, H. and Ohkubo, C. (2007) Effects of $12 \mathrm{mT}$ static magnetic field on sympathetic agonist-induced hypertension in Wistar rats. Bioelectromagnetics, 28, 369-378. doi:10.1002/bem.20307

[8] Kotani, H., Kawaguchi, H., Shimoaka, T., Iwasaka, M., Ueno, S., Ozawa, H., Nakamura, K. and Hoshi, K. (2002) Strong static magnetic field stimulates bone formation to a definite orientation in vitro and in vivo. Journal of Bone and Mineral Research, 17, 1814-1821. doi:10.1359/jbmr.2002.17.10.1814

[9] Singh, P., YashRoy, R. and Hoque, M. (2006) Augmented bone-matrix formation and osteogenesis under magnetic field stimulation in vivo TEM and SEM investigations. Indian Journal of Biochemistry and Biophysics, 43, 167-172.

[10] Xu, S., Okano, H., Tomita, N. and Ikada, Y. (2011) Recovery effects of a $180 \mathrm{mT}$ static magnetic field on bone mineral density of osteoporotic lumbar vertebrae in ovariectomized rats. Evidence-Based Complementary and Alternative Medicine 2011, online 620984.

[11] Segal, A.N., Toda, Y., Huston, J. and Saeki, Y. (2001) Two configurations of static magnetic fields for treating rheumatoid arthritis of the knee: A double-blind clinical trial. Archives of Physical Medicine and Rehabilitation, 82, 1453-1460. doi:10.1053/apmr.2001.24309

[12] Harlow, T., Greaves, C., White, A., Brown, L., Hart, A. and Ernst, E. (2004) Randomised controlled trial of magnetic bracelets for relieving pain in osteoarthritis of the hip and knee. British Medical Journal, 18, 1450-1454. doi:10.1136/bmj.329.7480.1450

[13] Chen, C.Y., Chen, C.L., Hsu, S.C., Chou, S.W. and Wang, K.C. (2008) Effect of magnetic knee wrap on quadriceps strength in patients with symptomatic knee osteoarthritis. Archives of Physical Medicine and Rehabilitation, 89, 2258-2264. doi:10.1016/j.apmr.2008.05.019

[14] Kanai, S., Taniguchi, N., Kawamoto, M., Endo, H. and Higashino, H. (2004) Effect of static magnetic field on pain associated with frozen shoulder. The Pain Clinic, 16, 173-179. doi:10.1163/156856904774134389

[15] Kanai, S. and Taniguchi, N. (2006) Effect of polarity exchangeable permanent magnet on frozen shoulder pain.
The Pain Clinic, 18, 37-45. doi: $10.1163 / 156856906775249811$

[16] Kanai, S., Taniguchi, N. and Okano, H. (2011) Effect of Magnetotherapeutic Device on Pain Associated With Neck and Shoulder Stiffness. Alternative Therapies in Health and Medicine, 17, 44-48.

[17] Matsuhata, I., Taniguchi, N. and Kanai, S. (2006) Study of Electro-Acupuncture Therapy (muscle-EAT) for adjuvant-induced arthritis in rats. Kanpo Medcine, 57, 437442.

[18] Kim, H.R., Rajaiah, R., Wu, Q.L., Satpute, S.R., Tan, M.T., Simon, J.E. and Berman, B.M. (2008) Green tea protects rats against autoimmune arthritis by modulating disease-related immune events. The Journal of Nutrition, 138, 2111-2116. doi: $10.3945 /$ jn. 108.089912

[19] Tamaki, K., Taniguchi, N., Matsuhata, I. and Kanai, S. (2005) Effects of moxibustion and gosya-zinnki-gann for spontaneous fatty diabetes rat. Kanpo Medcine, 56, 555560.

[20] Pekrkova, I., Parara, S., Holecek, V., Stopka, P., Trefil, L., Racek, J. and Rokyta, R. (2001) Does exogenous melatonin influence the free radicals metabolism and pain sensation in rat? Physiological Research, 50, 595-602.

[21] Kanai, S., Take, A. and Taniguchi, N. (2000) Study of moxibusition for experimental arthritis model rat. Oriental Medicine and the Pain Clinic, 30, 16-21.

[22] Omoto, A., Kawahito, Y., Prudovsky, I., Tubouchi, Y., Kimura, M., Ishino, H., Wada, M., Yoshida, M., Kohno, M., Yoshimura, R., Yoshikawa, T. and Sano, H. (2005) Copper chelation with tetrathiomolybdate suppresses adjuvant-induced arthritis and inflammation-associated cachexia in rats. Arthritis Research \& Therapy, 7, R1174R1182.

[23] Perera, P.K., Li, Y., Peng, C., Fang, W. and Han, C. (2010) Immunomodulatory activity of a Chinese herbal drug Yi Shen Juan Bi in adjuvant arthritis. Indian Journal of Pharmacology, 42, 65-69. doi:10.4103/0253-7613.64489

[24] Taniguchi, N., Kanai, S., Kawamoto, M., Endo, H. and Higashino, H. (2004) Study on application of static magnetic field for adjuvant arthritis rats. Evidence-Based Complementary and Alternative Medicine, 1, 187-192. doi:10.1093/ecam/neh024

[25] Takeshige, C. and Sato, M. (1996) Comparisons of pain relief mechanisms between needling to the muscle, static magnetic field, external qigong and needing to the acupuncture point. Acupuncture \& Electro-Therapeutics Research, 21, 119-131. 